\title{
Study of serum nitric oxide (no) in type 2 diabetes mellitus patients having normoalbuminuria and microalbuminuria in Central India
}

\author{
Jayshri Sadashiv Jankar ${ }^{1, *}$, Kumud Namdeorao Harley ${ }^{2}$, Kanchan Manoharrao Mohod ${ }^{3}$ \\ ${ }^{1}$ Assistant Professor, ${ }^{2,3}$ Associate Professor, Dept. of Biochemistry, ${ }^{1}$ BKL Walawalkar Rural Medical College, \\ Maharashtra, ${ }^{2}$ Govt. Medical College, Akola, Maharashtra, ${ }^{3}$ Mahatma Gandhi Institute of Medical Sciences, \\ Sewagram, Maharashtra, India
}

*Corresponding Author:

Email id: jayshrijankar@gmail.com

\begin{abstract}
Introduction: Nitric oxide (NO) is known as a mediator in a broad cluster of biological systems. NO is the endothelium-derived relaxing factor, which causes vasodilation by relaxing vascular smooth muscle. This role of NO plays an important role of diabetic nephropathy in Type 2 Diabetes Mellitus (DM)

Aims: To assess serum nitric oxide levels among type 2 diabetes mellitus patients having normoalbuminuria and microalbuminuria.

Setting and Design: This cross sectional study was carried out at Department of Biochemistry in a tertiary care rural hospital in Central India with 200 diagnosed patients of type 2 diabetes mellitus.

Materials and Methods: $24 \mathrm{hr}$ urine albumin was done in all the patients. Based on the concentration of albumin in urine, patients were categorised in two groups, group 1 had normoalbuminuria (i.e. Urine Albumin $<20 \mathrm{mg} / 24$ hr) and group 2 had microalbuminuria (i.e. Urine Albumin $>20$ to $<300 \mathrm{mg} / 24 \mathrm{hr}$ ). Then, serum NO was assessed and compared in these groups.

Results: Serum NO levels were significantly raised $(\mathrm{p}<0.05)$ in Group 2 as compared to Group 1.

Conclusion: Our observations suggest that serum NO levels measurement has a good detection potential for early stages of diabetic nephropathy manifested in the form of microalbuminuria in Type $2 \mathrm{DM}$
\end{abstract}

Keywords: Diabetic Nephropathy, Microalbuminuria, Nitric Oxide, Normoalbuminuria.

Received: $28^{\text {th }}$ August, 2017

Accepted: 05 ${ }^{\text {th }}$ October, 2017

\section{Introduction}

Diabetes, a chronic metabolic disorders characterized by hyperglycemia resulting from defects in insulin secretion, insulin action, or both. It is emerging as a major worldwide health problem with huge social and economic effects. ${ }^{1}$ In India, the incidence of diabetes is increasing rapidly. According to Wild et al. the prevalence of diabetes is predicted to double globally from 171 million in 2000 to 366 million in 2030 with a maximum increase in India $^{2}$ Type 2 Diabetes Mellitus (DM) which accounts for more 85-90\% of diabetic population and is more frequently neglected as a mild disease of elderly population. The mortality risk for individuals with diabetes is 2-3 times higher than the risk for people with normal glucose homeostasis. ${ }^{3}$ Long term diabetes and poor glycemic control also lead to several other seriously disabling disorders. Diabetic nephropathy is potential problem in both type $1 \mathrm{DM}$ and type $2 \mathrm{DM}$, one of the major causes of chronic renal failure in the west part of the world. ${ }^{4}$ It is well known fact that Microalbuminuria, an initial marker of diabetic nephropathy, is also an independent risk factor for cardiovascular disease. It is defined as urinary excretion of albumin in range of 30-300 $\mathrm{mg} / 24 \mathrm{hr}$; the increased urinary albumin excretion in urine may represent involvements of other systemic vascular endothelial damage than renal microvascular injury alone ${ }^{5}$ in type $2 \mathrm{DM}$, microalbuminuria or frank albuminuria may be present at the time of diagnosis, and microalbuminuria or incipient nephropathy more commonly accompanied by hypertension. Thus, microalbuminuria in patients with type $2 \mathrm{DM}$ is perhaps the most important early signal preceding the starting of systemic vasculopathies and associated with damage of target organs. Interestingly, damaged podocytes with excess production of, eventually extracellular release of ROS at the glomerular level can be caused by hyperglycemia, dyslipidemia and arterial hypertension manifesting in the form of albuminuria. ${ }^{6}$ Numerous studies demonstrated that hyperglycemia-induced generation of free radicals mediates oxidative stress which leads to the development and further progression of diabetes and periodically leads to vascular complications. $^{7-9}$

Depleted intracellular glutathione with an increased plasma extracellular superoxide dismutase are involved in lipid peroxidation and 
diabetic complications resulting from hyperglycemia induced oxidative stress. ${ }^{10-12}$ The diabetes associated endothelial dysfunction leads to decreased bioavailability of Nitric Oxide (NO) due to reduced NO synthesis from 1-arginine. New basic research provide possible explanations underlying the decreased NO bioavailability in type $2 \mathrm{DM}$. So, the nitric oxide is decreased in the progression of vascular disease like diabetes mellitus and hypertension. ${ }^{13,14}$

With this idea, we conducted the study to compare levels of serum NO in patients of type 2 DM having normoalbuminuria and microalbuminuria in a tertiary care hospital in central India.

\section{Materials and Methods}

This hospital based cross sectional study was conducted in a tertiary care hospital in Central India with 200 diagnosed patients of type 2 DM for more than 1 year, aged 30 years and above. These patients were categorised into 2 groups.

Group 1: Includes 123 subjects with Normoalbuminuria (i.e. Urine Albumin $<20 \mathrm{mg}$ / $24 \mathrm{hr}$ )

Group 2: Includes 77 subjects with Microalbuminuria (i.e. Urine Albumin $>20$ to $<300 \mathrm{mg} / 24 \mathrm{hr}$ )

In this study, we assayed levels of serum NO compared it in both the above groups.

\section{Method of Sample Collection}

The study was commenced after obtaining clearance from the Institutional Human Ethical Committee. After obtaining written/informed consent in regional language from Subject or Subject's legal representative, attending our tertiary level rural hospital,

1. Urine Sample: $24 \mathrm{hr}$ Urine Sample was collected to quantify albumin in urine

2. Blood Sample: $2 \mathrm{ml}$ of fasting blood sample was collected from anticubital vein in plain bulb. The serum was either analysed immediately for estimation of NO.

\section{Method of Analysis}

1. Single Radial Immuno Diffusion for Estimation of Microalbuminuria: ${ }^{15}$ Monospecific Human serum antibody $150 \mu \mathrm{l}$ (as standardized by trial and error method) was incorporated into $15 \mathrm{ml}$ of molten agarose gel $(1 \%)$ at $56^{\circ} \mathrm{C}$ taken in small glass beaker and mixed thoroughly by gentle rotation. The agarose gel was poured onto the glassslide of $12.5 \times 7.5 \mathrm{~cm}$ size. It was allowed to solidify for half an hour at $4^{\circ} \mathrm{C}$ in a humid chamber. Wells were punched out at $1.5 \mathrm{~cm}$ apart each other using the gel cutter and the water suction pump. The individual well was charged with $5 \mu$ of the urine/standard with micropipette. The loaded gel was allowed to diffuse for $24 \mathrm{hrs}$ at $4^{0} \mathrm{C}$ in a moist chamber. The resulting ring diameters of the circular immunoprecipitates were noted. Damp filter paper was kept over the slide for drying at room temperature. The dried plate was put into Coomassie brilliant blue $\mathrm{R}$ stain for 30 minutes. Then washed under the tap and destained with destaining solution till background was clear. Standard curve was plotted taking the square of the diameter $\left(D^{2}\right)$ on $y$ axis against the concentration of standards on $\mathrm{x}$ axis. Measurements of diameters of rings were used for reading the concentrations of the unknown samples.

2. Estimation of Nitric Oxide (NO)- Griess Reagent Assay: ${ }^{16}$ NO combines with oxygen very rapidly to form Nitrogen Dioxide $\left(\mathrm{NO}_{2}\right)$, so nitrite estimation in serum was done.

\section{Principle}

In acid solution, nitrite is converted into nitrous acid $\left(\mathrm{HNO}_{2}\right)$, which is diozolises sulphonamide. This sulphanilamide-dizonium salt is then reacted with $\mathrm{N}$-(1-Naphthyl)ethylenediamine (NED) to produce a chromophore, which is measured, colorimetrically, at 540nm.

\section{Reagents}

$2 \mathrm{mMol}$ Sodium Nitrite $\left(\mathrm{NaNO}_{2}\right)$ with Distilled water.

Griess reagent solution: Solution A-0.1\% Napthylethylene diamine dihydochloride: Solution B-1\% Sulfanilamide in $2.5 \%$ concentrated Phosphoric acid.

\section{Sample Preparation for Estimation of NO}

\begin{tabular}{|l|c|c|}
\hline & Test & Blank \\
\hline Griess reagent & $500 \mu \mathrm{l}$ & $500 \mu \mathrm{l}$ \\
\hline Serum & $50 \mu \mathrm{l}$ & - \\
\hline DW & $450 \mu \mathrm{l}$ & $500 \mu \mathrm{l}$ \\
\hline
\end{tabular}

Optical density measured by colorimetry, taken at $540 \mathrm{~nm}$ should be put in standard curve plot for obtaining $\mathrm{NO}$ concentration in $\mu \mathrm{M}$.

\section{Statistical Analysis}

Data analysis was done by using descriptive and inferential statistics using z-test for difference between two means, chi square test 
and Pearson's Correlation Coefficient, software used in the analysis were SPSS17.0 version and Graph Pad Prism 5.0 version and $\mathrm{p}<0.05$ was considered as significant \& p-value $>0.05$ was considered as non significant. All values were expressed as Mean \pm SD.

\section{Result}

This study was conducted among 200 diagnosed patients of type 2 DM aged more than
30 years with duration of disease more than 1 year. Patients were divided in to 2 groups; Group 1 included 123 patients having normoalbuminuria and group 2 included 77 patients microalbuminuria.

a. Distribution of Diabetic Subjects into Groups: Urine Albumin was done in all subjects. TYPE 2 DM subjects were grouped as Group 1 which is comprised of 123 subjects and Group 11 comprised of 77 as shown in Table 1.

Table 1: Distribution of Diabetic Patients into Groups

\begin{tabular}{|l|l|c|}
\hline Groups & Type 2 DM & Number of Patients (n) \\
\hline Group 1 & Normoalbuminuria & 123 \\
\hline Group 2 & Microalbuminuria & 77 \\
\hline
\end{tabular}

b. Comparision of Urine Albumin in both groups: On comparison of urine albumin in both groups, it was found to be significantly raised $(\mathrm{p}<0.05)$ in Group 2 as compared to Group 1 as seen Table 2

Table 2: Distribution of Diabetic Subjects into Groups

\begin{tabular}{|c|c|c|c|c|}
\hline & $\begin{array}{c}\text { Group 1 } \\
(n=123) \\
(\text { Mean } \pm \text { SD })\end{array}$ & $\begin{array}{c}\text { Group } 2 \\
(n=77) \\
(\text { Mean } \pm \text { SD })\end{array}$ & z-value & p-value \\
\hline $\begin{array}{l}\text { Urine Albumin } \\
\text { (mg/24hr) }\end{array}$ & $14.68 \pm 6.63$ & $60.76 \pm 18.73$ & 24.92 & $0.0001 \mathrm{~S}, \mathrm{p}<0.05$ \\
\hline
\end{tabular}

$\mathbf{n}=$ number; $\mathbf{S}=$ Significant

c. Comparision of serum Nitric Oxide levels in Group 1 \& Group 2: On comparing serum nitric oxide levels in both the study groups, it was observed that serum nitric oxide levels were significantly raised $(\mathrm{p}<0.05)$ in group 2 which denotes the progression of disease in the form of microalbuminuria.

Table 3: Comparision of serum Nitric Oxide in Group 1 \& Group 2

\begin{tabular}{|c|c|c|c|c|}
\hline \multirow{2}{*}{ Parameter } & Group I $(\mathbf{n = 1 2 3})$ & Group ll $(\mathbf{n}=\mathbf{7 7})$ & \multirow{2}{*}{ z-value } & \multirow{2}{*}{ p-value } \\
\cline { 2 - 3 } & Mean \pm SD & Mean \pm SD & & \\
\hline Nitric Oxide $(\boldsymbol{\mu M})$ & $1.54 \pm 0.27$ & $2.63 \pm 0.38$ & 23.61 & $0.0001, \mathrm{~S}$ \\
\hline
\end{tabular}

$\mathbf{n}=$ number; $\mathbf{S}=$ Significant

d. Duration wise status of serum Nitric Oxide $(\boldsymbol{\mu M})$ in both groups: Further, to find out the impact of duration of disease on the NO levels, we divided group 1 and group 2 subjects into $\leq 7$ years and $>7$ years duration of disease. As given in (Table 4), we observed significant increased level of $\mathrm{NO}$ with mean $\pm \mathrm{SD}$ of $1.84 \pm 0.23$ in group 1 with $>7$ years disease duration in comparision $\leq 7$ years with mean $\pm \mathrm{SD}$ of $1.42 \pm 0.19$. (p value $<0.05$ ). Further, NO levels were compared in Group 2 with duration of disease $>7$ years $\& \leq 7$ years, levels were found to be significantly low, in subjects with duration of disease $>7$ years in Group 2 .

Table 4: Duration wise status of serum Nitric Oxide $(\mu M)$ in both groups

$\mathbf{n}=$ number; $\mathbf{S}=$ Significant

\begin{tabular}{|l|c|c|}
\hline \multicolumn{1}{|c|}{ Duration } & Group 1 & Group 2 \\
\hline$\leq 7$ years & $1.42 \pm 0.19(\mathrm{n}=89)$ & $2.82 \pm 0.28(\mathrm{n}=51)$ \\
\hline$>7$ years & $1.84 \pm 0.23(\mathrm{n}=34)$ & $2.26 \pm 0.22(\mathrm{n}=26)$ \\
\hline p-value & $0.0001, \mathrm{~S}$ & $0.0001, \mathrm{~S}$ \\
\hline
\end{tabular}




\section{Discussion}

It is a well-studied fact that DM is a endangering factor for both for micro and macrovascular complications. ${ }^{17,18}$ Microvascular complications of diabetes include nephropathy, neuropathy and retinopathy, while macrovascular complications such as peripheral vascular disease, cerebrovascular disease and coronary artery disease are the most important causes of death in the diabetic population. Since, numerous studies demonstrated that oxidative stress, mediated mainly by hyperglycemia-induced generation of free radicals, contributes to the development and progression of diabetes and eventually leads to micro and macrovascular complications. ${ }^{19}$ Numerous studies have revealed the relationship of hyperglycemia induced oxidative stress leading to development and progression of diabetic complication through superoxide overproduction and the mitochondrial electron transport chain produced ROS. $^{20}$ Activation of protein kinase $\mathrm{C}$, increased flux through the hexosamine pathway and increased activity of the polyol pathway are directly caused by Reactive Oxygen Species (ROS). Other major effects of ROS are uncoupling of nitric oxide synthase, xanthine oxidase, NAD (P)H oxidase, and advanced. ${ }^{21}$ It is well known fact that there is NO alteration in diabetes and its associated complications. It easily reacts with superoxide, generating the highly reactive molecule known as peroxyl nitrite $\left(\mathrm{ONOO}^{-}\right)$, and triggering a cascade of harmful events. Microalbuminuria, which results in form of increased passage of albumin through the glomerular filtration, is an important predictor of progression of renal impairment and cardiovascular disease. Endothelial dysfunction proceeds of microalbuminuria in both types of diabetes. ${ }^{21}$ So, later manifestations of renal and cardiovascular diseases can be prevented by understanding the mechanism of microalbuminuria and designing therapies to treat them.

Present Cross Sectional study was conducted on 200 diagnosed patients of Type 2 DM which were categorised into 2 groups. (Table 1)

In this study, to detect the degree of renal impairment, we have studied urinary albumin excretion. (Table 2) Mean Urinary albumin level in Group 1 was $14.68 \mathrm{mg} / 24 \mathrm{hr}$ and in Group $1 \mathrm{l}$ it (mean urinary albumin level) was $60.70 \mathrm{mg} / 24 \mathrm{hr}$ which was found to be statistically significant. Microalbuminuria is significantly associated with nephropathy, and predictor for microvascular complications. Neil et al. $1993^{22}$ confirmed prediction given by microalbuminuria as a risk factor for morbidity and mortality in
Type 2 DM. In present study, it was found that microalbuminuria was increased significantly in Group 11 as compared to Group 1. As per J. Kanakamani et al. $2010^{23}$ prevalence of microalbuminuria in a hospital based study in Type 2 DM study was $24.7 \%$.

As shown in (Table 3), Serum nitric oxide levels were significantly raised in Group 11 in comparison to baseline Group 1. Present finding i.e. increased NO levels in microalbuminuric subjects (group II) is consistent with several previous works done till date. ${ }^{24,25,26}$ Although, our results showed significant increased levels of nitric oxide $(\mathrm{P}<0.0001)$ with mean $\pm \mathrm{SD}$ of 2.63 \pm 0.38 (p-vaue $=0.0001)$ in microalbuminuria group II in comparison with that of group I which includes normoalbuminuria diabetic subjects with mean \pm SD of NO levels $1.54 \pm 0.27,{ }^{27}$ in contrast, decreased levels were also reported by other investigators.229,230 The conflict among the results of previous studies might be due to geographical location and genetic background of the population. Smoking and food habit may also affect serum NO levels as reported previously by Das et al. ${ }^{28}$ According to recent literature published in 2015 which was conducted on Southern population in India have reported that hyperglycemia may enhance NO production through increased expression of nitric oxide synthase (NOS), eNOS (endothelial NOS), iNOS (inducible NOS) gene and protein levels. ${ }^{26}$ This hypothesis is supported by the continued observations, in both animal models and humans. Furthermore, several other studies also supported the finding that iNOS gene induction, in vitro and in vivo enhanced the production of reactive nitrogen species and increased protein nitrosylation. ${ }^{24,26,29,30}$ Maejima et al. in $2001^{31}$ suggested the possibility of its impaired action and compensatory product of $\mathrm{NO}$ against increased oxidant stress. Increased nitric oxide results thus may reflect the vascular injuries by increased oxidant stress and induced cytotoxic NO production via NOS activation. Higher NO levels in-vivo might have both beneficial as well as detrimental effect depending on the local environment of the system. On one hand, NO can cause relaxation of blood vessels and reduce hypertension, and thereby, act cytoprotective and on the other hand, NO may interact with superoxide radical $\left(\mathrm{O}_{2}^{-}\right)$leading to $\mathrm{NO}$ inactivation. The reaction of superoxide radical with NO is rapid and results in the formation of potent oxidant radical product called peroxynitrite $\left(\mathrm{ONOO}^{-}\right)$. This may contribute to impaired endothelial function by stimulating arachidonic acid metabolism, lipid peroxidation, and prostanoid production. ${ }^{32,33,34}$ 
Table 4 shows the impact of duration of disease on the NO levels. We observed significant increased level of $\mathrm{NO}$ with mean \pm $\mathrm{SD}$ of $1.84 \pm 0.23$ in group 1 with $>7$ years disease duration in comparision $\leq 7$ years with mean \pm SD of $1.42 \pm 0.19$. When NO levels were compared in Group 2 with duration of disease > 7 years $\& \leq 7$ years, levels were found to be significantly low, in subjects with duration of disease $>7$ years in Group 2. Observations from earlier study ${ }^{35}$ demonstrated the same finding which indicates endothelial dysfunction in later stages of diabetes which is consistent with our results.

Previous reports ${ }^{36,37}$ stated that, the bioavailability of NO has a vital role in vascular region of body. Activation of guanylyl cyclase on vascular smooth muscle cells caused by NO leads to vasodilation. In addition, protection of the blood vessel from endogenous injury is done by NO, in case of atherosclerosis; it is done by preventing platelet and leukocyte interaction with the vascular wall and inhibition of vascular smooth muscle cell proliferation and its migration. Conversely, decreased bioavailability endothelium-derived NO leads to increased activity of the proinflammatory transcription factor nuclear factor kappa B which results in expression of leukocyte adhesion molecules and production of various chemokines and cytokines. ${ }^{27}$ These actions result in promotion monocyte and vascular smooth muscle cell migration into the intima and ultimately formation of macrophage foam cells leading to the initial morphological changes of atherosclerosis. Thus, decreased bioavailability of NO in diabetes may underlie its predisposed atherosclerosis, ${ }^{26,28}$ production of NO via NOS and its degradation by oxygen-derived free radicals balances its bioavailability. Many of the metabolic derangements like hyperglycemia and liberation of excess free fatty acid which are common in diabetes affect the synthesis or degradation of NO by mediating abnormalities in endothelial cell function. Cell culture data with Human umbilical vein endothelial cells has confirmed $^{27}$ that high glucose exposure enhances NO production at early time point in diabetes but reduced subsequently after exposure for a longer duration. It is believed that decreased NO bioavailability was appeared to be associated with overproduction of superoxide and Larginine defeciency. All these changes may contribute to reduced concentration NO concentration and thereby increase risk for atherosclerosis. Decreased synthesis of NO might also result from abnormal handling of intracellular Calcium and consequent reduction in the activity of NOS. ${ }^{38}$

The present study finds support in the observation that diabetes affects nitric oxide metabolism as a successive and significant decrease was observed in the level of endothelial nitric oxide at the onset of diabetic complications, such as nephropathy. Several mechanisms could account for the low nitric oxide bioavailability in diabetes. 1) Glucose can search and collect the NO to form unspecified additional product, 2) Activation eNOS will be impaired, 3) oxidative stress suppresses NO to form peroxynitrite, 4) Endothelial NO defeciency may be resulted from formation of advanced glycation end (AGE) products in diabetes and finally, binding of NO to glycosylated deoxyhemoglobin may take place. ${ }^{38}$ Evidence suggest that resistances generated in glomerular arterioles are regulated by NO levels is supported by vasoconstriction in afferent and efferent arterioles of nephrons both superficial cortical (Lockhart et al., in 1994) and juxtamedullary region of kidneys (Zats, Nussi et al. in 1991) following inhibition of $\mathrm{NO}$ synthesis. ${ }^{39}$

\section{Conclusion}

In conclusion, higher NO level was associated with early stages of TYPE 2 DM. As we observed significant low NO level in TYPE 2 DM subjects with microalbuminuria group having duration of diabetes $>7 \mathrm{yrs}$, indicating that NO levels may alter with duration of disease and hence its estimation might be used as prognostic factor for predicting onset of vascular complications in this population. Various studies across the globe carry the massage that nitric oxide levels will soon be a diagnostic tool accessing the endothelium function.

\section{References}

1. Joanna M Tarr, Kirti Kaul, Mohit Chopra. Pathophysiology of Diabetic Retinopathy. ISRN Ophthalmology. 2013;p13

2. Wild S, Roglic G, Green A, Sicree R, King H. Global prevalence of diabetes-estimates for the year 2000 and projections for 2030. Diabetes Care. 2004;27(3):1047-53.

3. Barr EL, Zimmet PZ et al. Risk of cardiovascular and all-cause mortality in individuals with diabetes mellitus, impaired fasting glucose, and impaired glucose tolerance: the Australian Diabetes, Obesity, and Lifestyle Study (AusDiab). Circulation 2007;116:151-7.

4. Villar E, Chang SH et al. Incidences, treatments, outcomes, and sex effect on survival in patients 
with end-stage renal disease by diabetes status in Australia and New Zealand (1991 2005).

Diabetes Care 2007;30:3070-6.

5. Battisti WP, Palmisano J, Keane WE. Dyslipidemia in patients with type 2 diabetes: Relationships between lipids, kidney disease and cardiovascular disease. Clin Chem Lab Med 2003;41:1174-81

6. Nosadini R, Tonolo G. Blood glucose and lipid control as risk factors in the progression of renal damage in type 2 diabetes. J Nephrol 2003; 16:S42-7

7. B. Halliwell. J. Gutteridge, Free Radicals in Biology and Medicine, Oxford University Press, New York, NY, USA, 4th edition, 2007

8. K. B. Pandey, N. Mishra, and S. I. Rizvi, "Protein oxidation biomarkers in plasma of type 2 diabetic patients," Clinical Biochemistry. 2010;43( 45):508-11

9. Turko IV, Marcondes S, Murad F: Diabetesassociated nitration of tyrosine and inactivation of succinyl-CoA: 3-oxoacid CoA- transferase. Am J Physiol Heart Circ Physiol 2001, 281(6):H228994

10. Harris MI, Flegal KM, Cowie CC, Eberhardt MS, Goldstein DE, Little RR, et al. Prevalence of diabetes impaired fasting glucose and impaired glucose tolerance test. Diabetes

Care. 1998;21:518-24.

11. Lawrence M, Tierney, McPhee SJ, Papadakis MA. Current Medical diagnosis and treatment. New York: McGraw-Hill Medical; 2006. London: McGraw-Hill.

12. WHO 1998-Prevention and control of Diabetes Mellitus, Report of an Intercountry workshop, Dhaka, Bangladesh: 1998, SEA/NCD/40

13. James PE, Lang DT, Tufnell-Barret T, Milsom TA, Frenneaux MP. Vasorelaxation by red blood cells and impairment in diabetes. Circ Res. 2004;94:976-83

14. Sydow K, Mondon CE, Cooke JP. Insulin resistance: Potential role of the endogenous nitric oxide synthase inhibitor ADMA. Vasc Med. 2005;10:S35-43

15. Mancini G, Carbonana AO, Haremans JF, Immunochemical Quantitation of antigens by single radial immunodiffusion, 2:234-54, 1965

16. Lee DU Kang YJ et al. Nitric Oxide ASSAY, Effects of 13- Alkylsubstituted berberine alkaloids on the expression of COX-II, TNF-a, Inos AND IL-12 production in LPS stimulated macrophages. Life Sci.2003; 73:1401-12

17. Pyorala K, Laakso M, Uusitupa M: Diabetes and atherosclerosis: an epidemiologic view. Diabetes Metab Rev 1987, 3(2):463-524.

18. Laakso M: Hyperglycemia and cardiovascular disease in type 2 diabetes. Diabetes 1999, 48(5):937-42

19. W Todd Cade. Diabetes-Related Microvascular and Macrovascular Diseases in the Physical Therapy Setting. Phys Ther. 2008

Nov;88(11):1322-35

20. Ferdinando Giacco, Michael Brownlee. Oxidative stress and diabetic complications. Circ Res. 2010 Oct 29;107(9):1058-70.

21. S. C. Satchell, J. E. Tooke. What is the mechanism of microalbuminuria in diabetes: a role for the glomerular endothelium?. Diabetologia. 2008 May;51(5):714-25.

22. Neil A, Hawkins $M$ et al. A Prospective population-based study of microalbuminuria as a predictor of mortality in NIDDM. Diabetes Care 1993;7:996-03

23. J. Kanakamani, A.C. Ammini et al. Prevalence of Microalbuminuria Among Patients with Type 2 Diabetes Mellitus-A Hospital-Based Study from North India. Diabetes Technology \& Therapeutics. February 2010, 12(2):161-6

24. Cosentino F, Hishikawa K et al. High glucose increases nitric oxide synthase expression and superoxide anion generation in human aortic endothelial cells. Circulation. $1997 \mathrm{Jul}$ 1;96(1):25-8

25. Ramu Adela, Susheel Kumar Nethi et al. Hyperglycemia Enhances Nitric Oxide Production in Diabetes: A Study from South Indian Patients. PLOS ONE DOI:10.1371/journal.pone.0125270 April 20, 2015.

26. Yang P, Cao Y, Hua LI. Hyperglycemia induces iNOS gene expression and consequent nitrosative stress via JNK activation. Am J Obstet Gynecol. 2010;203(2):185.

27. Amrita G, Mingma LS et al. Serum nitric oxide status in patients with type 2 diabetes in Sikkim. Int J Appl Basic Med Res 2011;1(1):31-5.

28. Das L, Mishra A et al. Effect of smoke and nonsmoke tobacco on nitric oxide levels in diabetic patients.J Transl Intern Med. 2014;2:36-9.

29. Cai S, Khoo J, Channon KM. Augmented BH4 by gene transfer restores nitric oxide synthase function in hyperglycemic human endothelial cells. Cardiovascular Research.2005;65:823-31.

30. Zhang X, Fu Y et al. PERK pathway are involved in NO-induced apoptosis in endothelial cells cocultured with RPE under high glucose conditions. Nitric Oxide. 2014;40C:10-6.

31. Maejima K, Nakano S et al. Increased basal levels of plasma nitric oxide in Type 2 diabetic subjects. Relationship to microvascular complications. J Diabetes Complications. 2001 MayJun;15(3):135-43.

32. Evans JL, Goldfine ID, Maddux BA, Grodsky GM: Oxidative stress and stress-activated signaling pathways: a unifying hypothesis of type 2 diabetes. Endocr Rev 2002, 23(5):599-622.

33. Francesco $\mathrm{C}$, Keiichi $\mathrm{H}$ et al. High glucose increases nitric oxide synthase expression and superoxide anion generation in human aortic endothelial cells. Circulation.1997;96:25-8.

34. Naohito I, Kaushik PP et al. (2001) Nitric oxide synthesis and oxidative stress in the renal cortex of rats with diabetes mellitus. J Am Soc Nephrol.2001;12:1630-19.

35. Valiatti FB, Crispim Det al.The role of vascular endothelial growth factor in angiogenesis and diabetic retinopathy. Arq Bras Endocrinol Metabol 2011, 55(2):106-13.

36. Schechter AN, Gladwin MT. Hemoglobin and the paracrine and endocrine functions of nitric oxide. N Engl J Med. 2003;348:1483-5.

37. Forstermann U, Munzel T. Endothelial nitric oxide synthase in vascular disease: from marvel to menace. Circulation. 2006;113:1708-14. 
38. Nakagawa T, Segal M, Croker B, Johnson RJ: A breakthrough in diabetic nephropathy: the role of endothelial dysfunction. Nephrol Dial Transplant 2007, 22(10):2775-7.

39. Eman Y. Mohammed, Dhia J. Al-Timim et al. Serum Nitric Oxide as a Biochemical Marker for Diabetic Nephropathy. Journal of Advanced Biomedical \& Pathobiology Research.2014;4(1),16-26. 